\title{
Serum Cholesterol Levels at the Onset of Bloodstream Infection Have Prognostic Value
}

\author{
Takatoshi Kitazawa $^{1,2}$, Shintaro Yanagimoto ${ }^{1}$, Keita Tatsuno ${ }^{1}$, Atsuhito Fukushima ${ }^{1}$, Shu Okugawa ${ }^{1}$, \\ Yasuo Ota ${ }^{2}$ \\ ${ }^{1}$ Department of Infectious Diseases, Graduate School of Medicine, The University of Tokyo, Tokyo, Japan; ${ }^{2}$ Department of Medicine, \\ Teikyo University, Tokyo, Japan. \\ Email: tkitazaw@med.teikyo-u.ac.jp
}

Received August $16^{\text {th }}, 2012$; revised September $18^{\text {th }}, 2012$; accepted October $19^{\text {th }}, 2012$

\begin{abstract}
Objective: The aims of this study were to investigate the changes in serum cholesterol levels at the onset of bloodstream infection (BSI) and to determine whether serum cholesterol levels were associated with patients' clinical backgrounds and the prognosis of BSI. Methods: A retrospective chart review was done to collect demographic information and the subjects' medical history, invasive procedures, and medications. Patients and Methods: From April 2003 to March 2006, all patients aged $\geq 20$ years with positive blood cultures in the University of Tokyo Hospital (a tertiary teaching hospital with 1200 beds) were enrolled. Results: Average cholesterol levels before the onset of BSI were 166.5 $\pm 46.5 \mathrm{mg} / \mathrm{dL}$, and, at the onset of BSI, they decreased to $134.4 \pm 45.0 \mathrm{mg} / \mathrm{dL}(\mathrm{p}<0.001)$. Thirty-day survivors had higher cholesterol levels both before and at the onset of BSI than non-survivors. Cholesterol levels at the onset of BSI were associated with the organisms of BSI. Patients with lower cholesterol levels tended to have higher 30-day mortality rates and longer medical treatment than patients with higher cholesterol levels ( $13 \%$ vs $3 \%, p=0.12 ; 24.3$ days vs 18.4 days, $p=$ 0.15). Conclusion: Cholesterol levels at the onset of BSI could be used as a prognostic marker in patients with BSI.
\end{abstract}

Keywords: Bloodstream Infection; Cholestereol; Prognosis

\section{Introduction}

Infection and inflammation induce the acute phase response, which leads to multiple changes in lipid metabolism. In various types of acute tissue injury, such as myocardial infarction, the plasma cholesterol level is consistently depressed [1]. It was previously reported that, over the clinical course of severe sepsis, plasma cholesterol levels rapidly decreased and then increased slowly [2]. However, the cholesterol levels prior to the onset of sepsis were not reported. To the best of our knowledge, there have been no reports dealing with the changes in serum cholesterol levels from the pre-clinical stage of bloodstream infection (BSI) to the onset of BSI. A previous study reported that lower plasma cholesterol levels were observed in patients with sepsis caused by gram-positive cocci or gram-negative rods [2]. In addition, hypocholesterolemia has been reported to be a prognostic marker in patients with acute inflammation, such as febrile neutronpenia and critically ill surgical patients $[3,4]$. Recently, low level of apolipoprotein A-I is associated with a marked impairment of effective arterial volume, multiple organ dysfunction and a poor prognosis [5-7]. However, no previous studies have determined whether serum choles- terol levels and their changes at the onset of BSI are associated with the causative organisms of BSI and the prognosis of BSI. In the present study, we investigated whether serum cholesterol levels changed at the onset of BSI, and whether serum cholesterol levels were associated with the pathogens and the clinical outcome of BSI.

\section{Patients and Methods}

\subsection{Patients}

From April 2003 to March 2006, all patients aged $\geq 20$ years with positive blood cultures in the University of Tokyo Hospital (a tertiary teaching hospital with 1200 beds) were enrolled. A retrospective chart review was done to collect demographic information and the subjects' medical history, invasive procedures, and medications. This study was approved by the review boards of the University of Tokyo.

\subsection{Data Collection and Definition}

Patients whose serum cholesterol levels were obtained during both of the following periods were included: the first period lasted between $10-30$ days before the onset 
of BSI (as a baseline cholesterol level); the second period lasted between 0 - 2 days after the onset of BSI. Patients for whom lipid lowering drugs were prescribed were excluded. Total cholesterol levels were determined using an autoanalyzer (Hitachi 7600-120; Hitachi-medico, Tokyo, Japan). Patients whose CRP levels at the first period were greater than $1.0 \mathrm{mg} / \mathrm{dL}$ were excluded from the study. Patients who had more than one episode of BSI caused by the same pathogen within 30 days after the previous episode were regarded as having the same episode. The patients were divided into two groups according to their total cholesterol levels both before and at the onset of bacteremia; the average total cholesterol levels before and at the onset of BSI were used to divide the groups. The onset of BSI was defined as the time when positive blood cultures were collected. The CRP at the onset of BSI was defined as the maximum CRP that was recorded during the first three $(0$ - 2) days after the onset of BSI. The severity of BSI was assessed using the Acute Physiology and Chronic Health Evaluation II (APACHE II) scores [8].

\subsection{Microbiology}

Blood specimens were inoculated using BacT/ALERT FAN bottles (BioMerieux); the positivity of blood cultures was judged using the BacT/ALERT 3D system (BioMerieux), the automated microbial detection system using colorimetric sensor-and-detection technology which detects microorganisms by tracking $\mathrm{CO}_{2}$ production.

\subsection{Statistical Analysis}

Relationships between categorical variables were analyzed using Pearson's chi-square test; Fisher's exact test was used when the expected count was less than 5. Continuous variables were compared using Student's $t$-test. All $\mathrm{p}$ values were two-sided, and $\mathrm{p}<0.05$ was considered significant. All of the analyses were performed using SPSS software for Windows (Ver.10.1).

\section{Results}

\subsection{Demographic Data of Patients with BSI}

Of the 1667 cases with positive blood cultures during the 3 years of surveillance, 81 met the criteria in that their cholesterol levels were examined both before and at the onset of BSI (Table 1). Fifty-eight patients were male (72\%), and 23 were female $(28 \%)$. The average age of the patients was $61 \pm 16$ years (range, $21-96$ years). With respect to preexisting comorbidities, $37(46 \%)$ patients had malignancies. The numbers of each pathogens of BSI were as follows; Streptococcus spp. in seven, Staphylococcus aureus in five, Coagulase-negative staphylococci in 11, Enterococcus spp. in six, Escherichia coli in six,
Table 1. Demographic data of BSI patients.

\begin{tabular}{cc}
\hline Charasteristics & value \\
\hline Age (year) & $61(21-96)$ \\
Average (range) & $58 / 23$ \\
Sex (male/female) & \\
Underlying disease & \\
Malignancy & 10 \\
Hematological & 27 \\
Nonhematological & 5 \\
Collagen disease & \\
Chronic viral & 9 \\
hepatitis, liver cirrhosis & 3 \\
Diabetes & 3 \\
Coronary heart disease & 5 \\
Cerebrovascular disease & \\
Others & \\
Total & \\
\hline
\end{tabular}

Klebsiella pneumoniae in three, Enterobacter spp. in six, Serratia spp. in two, Proteus spp. in two, Citrobacter spp. in three, Pseudomonas aeruginosa in three, other bacterium in 13, Candida spp. in five, other fungus in one, and polymicrobial in 11 .

\subsection{Cholesterol Levels before and at the Onset of BSI}

First, changes in serum total cholesterol levels before and at the onset of BSI were examined. Serum total cholesterol levels before and at the onset of BSI are shown in Figure 1. The average total cholesterol level before the onset of BSI was $166.5 \pm 46.5 \mathrm{mg} / \mathrm{dL}$; it decreased significantly to $134.4 \pm 45.0 \mathrm{mg} / \mathrm{dL}$ at the onset of BSI ( $p<$ 0.001). These results indicated that, at the onset of BSI, total cholesterol levels decreased from the baseline cholesterol levels.

In five patients the cholesterol level was elevated at the onset of BSI by more than $10 \mathrm{mg} / \mathrm{dL}$, and in eight patients the change of the cholesterol levels between before BSI and at the onset of BSI were within $10 \mathrm{mg} / \mathrm{dL}$. There was no difference between the non-cholesterol decreasing group and the cholesterol non-decreasing group in age, sex, underlying diseases, and type of pathogens. One patient with the elevated cholesterol level at the onset died within 30 days. 


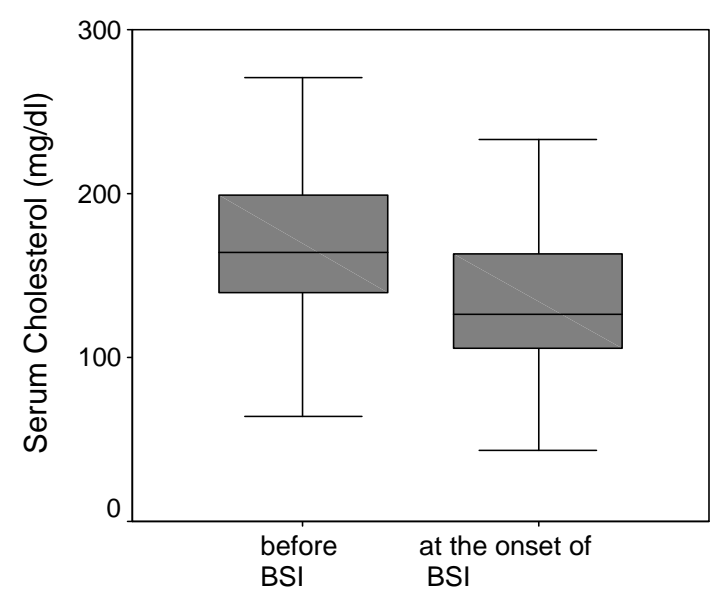

Figure 1. Changes in total cholesterol levels between before and at the onset of BSI. Boxplot analyisis of total cholesterol levels before and at the onset of BSI was performed. Box encloses central 2 quartiles of the data. A line in the box shows the average of the data.

\subsection{Serum Total Cholesterol Levels in Survivors and Non-Survivors}

Serum total cholesterol levels before and at the onset of BSI were compared between 30-day survivors and nonsurvivors. Total cholesterol levels before BSI were significantly lower in non-survivors than in survivors $(\mathrm{p}=$ 0.032) (Table 2). Total cholesterol levels at the onset of BSI were also significantly lower in non-survivors than in survivors $(p=0.049)$. However, the changes in cholesterol levels from before BSI to the onset of BSI were not different between non-survivors and survivors. These results show that non-survivors had lower total cholesterol levels than survivors both before and at the onset of BSI.

\subsection{Cholesterol Levels before the Onset of BSI Are Not Associated with Clinical Outcomes in Patients with BSI}

The patients were divided into two groups according to their serum total cholesterol levels before the onset of BSI. The average serum cholesterol level before the onset of BSI $(167 \mathrm{mg} / \mathrm{dL})$ was used as the cut-off point. As shown in Table 3, the clinical backgrounds and causative organisms were not different between the two groups. In addition, no significant differences between the two groups were observed in 30-day mortality and average treatment durations. These results suggest that serum total cholesterol levels before the onset of BSI were not associated with clinical outcomes in patients with BSI.

\subsection{Cholesterol Levels at the Onset of BSI Are a Prognostic Marker of BSI}

Finally, whether serum total cholesterol levels at the onset of BSI could be used as a prognostic indicator of BSI was examined. The patients were divided into two groups according to their serum total cholesterol levels at the onset of BSI. The average cholesterol level at the onset of BSI $(134 \mathrm{mg} / \mathrm{dL})$ was used as the cut-off. As shown in Table 4, there were no significant differences in clinical backgrounds (age, sex, proportion of patients with malignancy, CRP levels, and proportion of patients with an APACHE II score >15) between the two groups. However, in the higher cholesterol group, gram-positive cocci were more frequently detected than gram-negative rods, while gram-negative rods were the dominant causative organisms in the lower cholesterol group. In fact, the ratio of gram-positive cocci to gram-negative rod infections was significantly different between the two groups $(\mathrm{p}=0.05)$. Thirty-day mortality tended to be higher in the lower cholesterol group (13\%) than in the higher cholesterol group $(3 \%)$, though the difference was not significant $(p=0.12)$. Furthermore, the average treatment duration was longer in the lower cholesterol group than in the higher cholesterol group, though the difference was not significant $(\mathrm{p}=0.15)$.

In order to evaluate the cholesterol level with the lowest false-negative rate in the 30-day mortality, other cutoff levels at the onset of BSI were selected instead of the average cholesterol level. Using a serum cholesterol cutoff value of $143 \mathrm{mg} / \mathrm{dL}$ at the onset of BSI, which was the highest level in non-survivors, the lower cholesterol group had a significantly higher mortality than the higher cholesterol group $(14 \%$ vs $0 \% ; p=0.04)$. The lower cholesterol group had a sensitivity of $100 \%$, a specificity of $39 \%$, a false-positive rate of $61 \%$, and a false-negative rate of $0 \%$ with respect to 30-day mortality. Receiver Operating Characteristic (ROC) curve analysis was performed (Figure 2); the area under the ROC curve (AUC) was 0.66 . These results imply that patients with lower serum total cholesterol levels at the onset of BSI have a worse prognosis than those with higher serum total cholesterol levels. Thus, the serum total cholesterol level at the onset of BSI could be used as a prognostic marker for BSI.

\section{Discussion}

In the present study, the cholesterol levels obtained before and at the onset of BSI were compared retrospectively. It was found the total cholesterol levels decreased significantly at the onset of BSI. A previous report involving 17 patients with severe sepsis found that total cholesterol levels decreased and reached a nadir on day 3; however, this study did not report the total cholesterol levels prior to the onset of sepsis [2]. This previous report also showed that total cholesterol levels decreased in patients with severe sepsis as a result of decreases in both LDL cholesterol and HDL cholesterol levels [2]; however, the mechanisms responsible for the decreases in the 
Table 2. Comparison of the cholesterol level before BSI and at the onset of BSI between non-survivors and surviviors.

\begin{tabular}{cccc}
\hline & $\begin{array}{c}\text { Non-survivors } \\
(\mathrm{n}=7)\end{array}$ & $\begin{array}{c}\text { Survivors } \\
(\mathrm{n}=74)\end{array}$ & $\mathrm{p}$ \\
\hline Tolal cholesterol level before BSI (mg/dL) & $130 \pm 45$ & $170 \pm 46$ & 0.032 \\
Tolal cholesterol level at the onset of BSI (mg/dL) & $103 \pm 40$ & $138 \pm 45$ & 0.049 \\
Change in total cholesterol level (mg/dL) & $-27 \pm 48$ & $-32 \pm 37$ & 0.84 \\
Change in total cholesterol level (\%) & $-12.3 \pm 54.4$ & $-17.3 \pm 22.6$ & 0.81 \\
\hline
\end{tabular}

Data are shown as the average cholesterol levels \pm standard deviation.

Table 3. Comparison of host factors and pathogens between low cholesterol group and high cholesterol group before BSI.

\begin{tabular}{|c|c|c|c|}
\hline & $\begin{array}{c}\text { Lower cholesterol } \\
\text { (Total cholesterol }<167 \mathrm{mg} / \mathrm{dl} \text { ) } \\
(\mathrm{n}=44)\end{array}$ & $\begin{array}{c}\text { Higher cholesterol } \\
\text { (Total cholesterol } \geq 167 \mathrm{mg} / \mathrm{dl}) \\
(\mathrm{n}=37)\end{array}$ & $\mathrm{p}$ \\
\hline Age & $62(21-96)$ & $60(23-87)$ & 0.62 \\
\hline Males & $31(70 \%)$ & $27(77 \%)$ & 0.80 \\
\hline \multicolumn{4}{|l|}{ Perexisting comorbidity } \\
\hline Malignancy & $20(45 \%)$ & $17(49 \%)$ & 0.96 \\
\hline Pathogens & & & 0.85 \\
\hline Gram-positive cocci & $16(36 \%)$ & $15(41 \%)$ & \\
\hline Gram-negative rods & $17(39 \%)$ & $13(35 \%)$ & \\
\hline Other bacteria & $3(7 \%)$ & $1(3 \%)$ & \\
\hline Fungus & $2(5 \%)$ & $3(9 \%)$ & \\
\hline Coinfection & $6(14 \%)$ & $5(15 \%)$ & \\
\hline $\mathrm{CRP}$ at the onset (mg/dl) & 10.0 & 12.2 & 0.21 \\
\hline APACHE II $>15$ at the onset & $11(25 \%)$ & $8(18 \%)$ & 0.14 \\
\hline 30 day mortality & $5(11 \%)$ & $2(6 \%)$ & 0.34 \\
\hline Treatment duration (days) & $\begin{array}{c}20.7 \pm 15.7 \\
(\mathrm{n}=37)\end{array}$ & $\begin{array}{c}19.7 \pm 16.1 \\
(\mathrm{n}=33)\end{array}$ & 0.88 \\
\hline
\end{tabular}

Data of age and treatment duration are shown as the average age \pm standard deviation. Other data are shown as the numbers of male patients, patients who have malignancy, patients who had BSI with each pathogens, respectively.

Table 4. Comparison of host factors and pathogens between low cholesterol group and high cholesterol group at the onset of BSI.

\begin{tabular}{|c|c|c|c|}
\hline & $\begin{array}{c}\text { Lower cholesterol } \\
\text { (Total cholesterol }<134 \mathrm{mg} / \mathrm{dl}) \\
(\mathrm{n}=47)\end{array}$ & $\begin{array}{c}\text { Higher cholesterol } \\
\text { (Total cholesterol } \geq 134 \mathrm{mg} / \mathrm{dl}) \\
(\mathrm{n}=34)\end{array}$ & $\mathrm{p}$ \\
\hline Age & $64(26-96)$ & $58(21-87)$ & 0.08 \\
\hline Males & $36(82 \%)$ & $22(65 \%)$ & 0.15 \\
\hline \multicolumn{4}{|l|}{ Perexisting comorbidity } \\
\hline Malignancy & $24(51 \%)$ & $13(38 \%)$ & 0.25 \\
\hline Pathogens & & & 0.05 \\
\hline Gram-positive cocci & $13(28 \%)$ & $18(53 \%)$ & \\
\hline Gram-negative rods & $20(43 \%)$ & $10(29 \%)$ & \\
\hline Other bacteria & $4(9 \%)$ & $0(0 \%)$ & \\
\hline Fungus & $3(6 \%)$ & $2(6 \%)$ & \\
\hline Coinfection & $7(15 \%)$ & $4(11 \%)$ & \\
\hline APACHE II $>15$ at the onset & $9(19 \%)$ & $10(29 \%)$ & 0.36 \\
\hline 30 day mortality & $6(13 \%)$ & $1(3 \%)$ & 0.12 \\
\hline Treatment duration (days) & $\begin{array}{c}24.3 \pm 26.5 \\
(n=30)\end{array}$ & $\begin{array}{c}18.4 \pm 36.7 \\
(n=31)\end{array}$ & 0.15 \\
\hline
\end{tabular}

Data of age and treatment duration are shown as the average age \pm standard deviation. Other data are shown as the numbers of male patients, patients who have malignancy, patients who had BSI with each pathogens, respectively. 


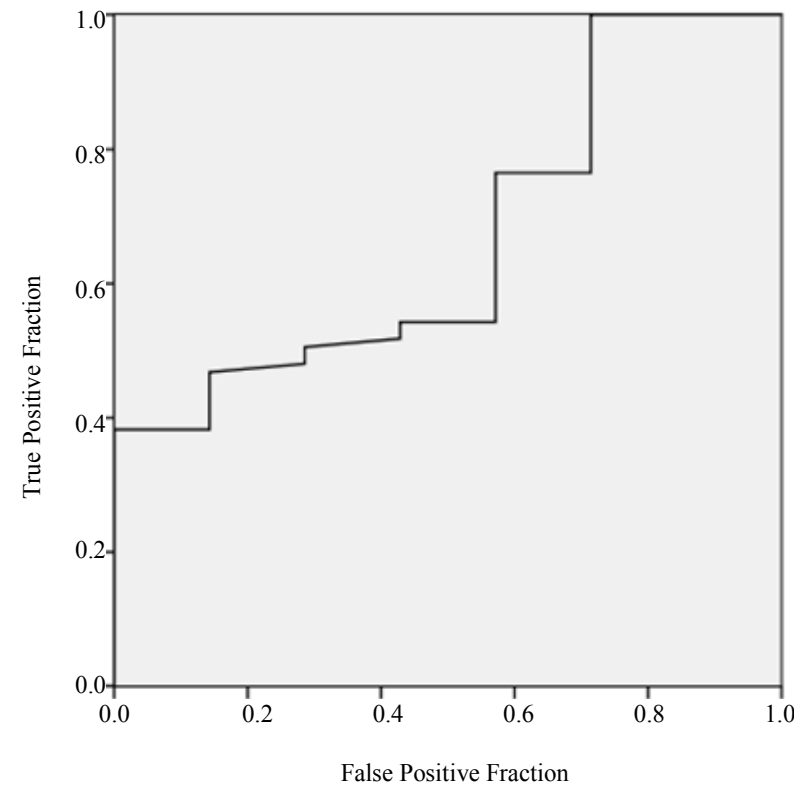

Figure 2. Receiver operating characteristic (ROC) curves of prognosis prediction based on the cholesterol level at the onset of BSI.

cholesterol levels have not been thoroughly studied. All of the 81 patients in our study had their cholesterol levels measured before the onset of BSI and at the onset of BSI; however, the number of patients whose HDL cholesterol and LDL cholesterol levels were available was small due to the retrospective nature of the data collection. Therefore, changes in LDL and HDL cholesterol levels during BSI were not examined in the present study.

Total cholesterol levels before BSI were significantly lower in non-survivors than survivors (Table 2), whereas between the two groups devided according the total cholesterol levels before BSI, no significant difference was observed in 30-day mortality (Table 3). Two non-survivors were classified into the higher group before BSI, although the cholesterol levels of the two non-survivors were slightly higher than the cutoff of $169 \mathrm{mg} / \mathrm{dL}$. The reason why non-survivors had lower cholesterol levels before BSI has not been elucidated in this study. Lipoproteins bind to and neutralize lipopolysaccharide (LPS) [9]. HDL reduced LPS-induced activation of coagulation and fibrinolysis and collagen stimulated platelet aggregation [10]. Cholesterol also has a central role in the production of adrenal hormones, primarily cortisol [11]. Furthermore, hypocholesterolemia is related to some plasma proteins and iron binding capacity [12]. We speculated that these factors may reduce the immunological function in hypocholesterolemic patients, and induce poor prognosis in bacteremia.

The present study also found that the causative organisms differed in the higher and lower cholesterol groups at the onset of BSI; gram-negative rods were more fre- quent pathogens in the lower cholesterol group. No previous reports have dealt with the relationship between serum cholesterol levels and the type of causative organisms in BSI. Various pathogenic components of the microorganisms are known to induce inflammatory cytokines. In a study of patients with sepsis, it was found that patients with gram-negative bacterial infections had higher TNF- $\alpha$ levels than those with gram-positive bacterial infections $[13,14]$. Furthermore, in a study comparing patients with septic shock to healthy controls, a significant inverse correlation was observed between serum TNF and serum cholesterol levels [15]. Given these reports and the results of the present study, the decrease in cholesterol levels at the onset of BSI may be due to inflammatory cytokines induced by microbial infection, and the different changes in cholesterol levels induced by different pathogens may be due to the degree of inflammatory cytokines induced by pathogenic components.

Furthermore, 30-day mortality tended to be higher in patients with lower cholesterol levels at the onset of BSI $(12 \%)$ than in patients with higher cholesterol levels (3\%, $\mathrm{p}=0.10$ ). It was also found that hypocholesterolemia at the onset of BSI was associated with prolonged treatment duration. In assessing the 30-day mortality of BSI, the cholesterol level with the lowest false-negative rate was considered to be the most valuable. Using a serum cholesterol cutoff value of $143 \mathrm{mg} / \mathrm{dL}$ at the onset of BSI, the lower cholesterol group had a false-negative rate of $0 \%$ with respect to 30 -day mortality. Given these results, serum total cholesterol levels at the onset of BSI could be used as a clinical prognostic indicator. We suggested that in suspected cases of BSI, total cholesterol should be measured at the onset of the illnesses, and patients whose cholesterol levels were lower than $143 \mathrm{mg} / \mathrm{dL}$ should be treated immediately with antibiotic therapy.

Our study had some limitations. The retrospective design of this study could have led to data loss; there is also the possibility of measurement bias in certain variables. The study was conducted at a single institution; the clinical backgrounds of BSI patients may be different in other institutions. However, it was appropriate to limit our investigation to patients treated in a single institution so as to identify changes that occurred at the onset of BSI. Most patients analyzed in this study were Japanese. Although no studies have dealt with racial differences in total cholesterol level changes during sepsis, differences might exist. In conclusion, patients develop hypocholesterolemia at the onset of BSI, and the serum total cholesterol level could be used as a prognostic marker for 30-day mortality and treatment duration.

In conclusion, low cholesterol levels at the onset of BSI could be used as a poor prognostic marker in patients with BSI. In suspected cases of BSI, total cholesterol should be measured at the onset of the illnesses. 


\section{Acknowledgements}

We are grateful to Mie Kataoka for preparing this manuscript.

\section{REFERENCES}

[1] R. S. Rosenson, "Myocardial Injury: The Acute Phase Response and Lipoprotein Metabolism," Journal of the American College of Cardiology, Vol. 22, No. 3, 1993, pp. 933-940. doi:10.1016/0735-1097(93)90213-K

[2] H. J. van Leeuwen, E. C. Heezius, G. M. Dallinga, J. A. van Strijp, J. Verhoef and K. P. van Kessel, "Lipoprotein Metabolism in Patients with Severe Sepsis," Critical Care Medicine, Vol. 31, No. 5, 2003, pp. 1359-1366. doi:10.1097/01.CCM.0000059724.08290.51

[3] P. Fraunberger, J. Hahn, E. Holler, A. K. Walli and D. Seidel, "Serum Cholesterol Levels in Neutropenic Patients with Fever," Clinical Chemistry and Laboratory Medicine, Vol. 40, No. 3, 2002, pp. 304-307. doi:10.1515/CCLM.2002.048

[4] B. R. Gordon, T. S. Parker, D. M. Levine, S. D. Saal, J. C. Wang, B. J. Sloan, P. S. Barie and A. L. Rubin, "Low Lipid Concentrations in Critical Illness: Implications for Preventing and Treating Endotoxemia," Critical Care Medicine, Vol. 24, No. 4, 1996, pp. 584-589. doi:10.1097/00003246-199604000-00006

[5] M. H. Tsai, Y. S. Peng, Y. C. Chen, J. M. Lien, Y. C. Tian, J. T. Fang, H. H. Weng, P. C. Chen, C. W. Yang and C. S. Wu, "Low Serum Concentration of ApolipoProtein A-I Is an Indicator of Poor Prognosis in Cirrhotic Patients with Severe Sepsis," Journal of Hepatology, Vol. 50, No. 5, 2009, pp. 906-915. doi:10.1016/j.jhep.2008.12.024

[6] J. Y. Chien, J. S. Jerng, C. J. Yu and P. C. Yang, "Low Serum Level of High-Density Lipoprotein Cholesterol Is a Poor Prognostic Factor for Severe Sepsis," Critical Care Medicine, Vol. 33, No. 8, 2005, pp. 1688-1693. doi:10.1097/01.CCM.0000171183.79525.6B

[7] C. Chenaud, P. G. Merlani, O. Bandshapp and B. Ricou, "Serum Lipids or Apolipoprotein A-I and Disease Severity of Meningococcal Sepsis," Critical Care Medicine, Vol. 34, No. 1, 2006, pp. 270-271.

\section{doi:10.1097/01.CCM.0000191256.88821.28}

[8] W. A. Knaus, E. A. Draper, D. P. Wagner and J. E. Zimmerman, "APACHE II: A Severity of Disease Classification System," Critical Care Medicine, Vol. 13, No. 10, 1985, pp. 818-829. doi:10.1097/00003246-198510000-00009

[9] D. M. Levine, T. S. Parker, T. M. Donnelly, A. Walsh and A. L. Rubin, "In Vivo Protection against Endotoxin by Plasma High Density Lipoprotein," Proceedings of the $\mathrm{Na}$ tional Academy of Sciences (USA), Vol. 90, No. 24, 1993, pp. 12040-12044. doi:10.1073/pnas.90.24.12040

[10] D. Pajkrt, P. G. Lerch, T. van der Poll, M. Levi, M. Illi, J. E. Doran, B. Arnet, A. van den Ende, J.W. Ten Cate and S. J. van Deventer, "Differential Effects of Reconstituted High-Density Lipoprotein on Coagulation, Fibrinolysis and Platelet Activation during Human Endotoxemia," Journal of Thrombosis and Haemostasis, Vol. 77, No. 2, 1997, pp. 303-307.

[11] P. Vyroubal, C. Chiarla, I. Giovannini, R. Hyspler, A. Ticha, D. Hrnciarikova and Z. Zadak, "Hypocholesterolemia in Clinically Serious Conditions-Review," Biomedical Papers, Vol. 152, No. 2, 2008, pp. 181-189. doi: $10.5507 /$ bp.2008.029

[12] C. Chiarla, I. Giovannini and J. H. Siegel, "The Relationship between Plasma Cholesterol, Amino Acids and Acute Phase Proteins in Sepsis," Amino Acids, Vol. 27, No. 1, 2004, pp. 97-100. doi:10.1007/s00726-004-0064-x

[13] J. Cohen and E. Abraham, "Microbiologic Findings and Correlations with Serum Tumor Necrosis Factor-Alpha in Patients with Severe Sepsis and Septic Shock," International Journal of Infectious Diseases, Vol. 180, No. 1, 1999, pp. 116-121. doi:10.1086/314839

[14] P. Kragsbjerg, H. Holmberg and T. Vikerfors, "Dynamics of Blood Cytokine Concentrations in Patients with Bacteremic Infections," Scandinavian Journal of Infectious Diseases, Vol. 28, No. 4, 1996, pp. 391-398. doi: $10.3109 / 00365549609037926$

[15] P. Fraunberger, G. Pilz, P. Cremer, K. Werdan and A. K. Walli, "Association of Serum Tumor Necrosis Factor Levels with Decrease of Cholesterol during Septic Shock," Shock, Vol. 10, No. 5, 1998, pp. 359-363. doi:10.1097/00024382-199811000-00009 